\title{
Modeling magnetic saturation and saliency effects via Euler-Lagrange models with complex currents for three-phase permanent magnet machines
}

\author{
D. Basic ${ }^{*}$ F. Malrait ${ }^{* *}$ P. Rouchon ${ }^{* * *}$ \\ * Schneider Electric, STIE, 33, rue André Blanchet, 27120 Pacy-sur \\ Eure.E-mail: duro.basic@schneider-electric.com \\ ** Schneider Electric, STIE, 33, rue André Blanchet, 27120 Pacy-sur \\ Eure. E-mail:francois.malrait@schneider-electric.com \\ *** Mines ParisTech, Centre Automatique et Systèmes, Mathématiques \\ et Systèmes, 60 Bd Saint-Michel, 75272 Paris cedex 06, France. \\ E-mail: pierre.rouchon@mines-paristech.fr
}

\begin{abstract}
:
Permanent magnet machines with both magnetic saturation and saliency effects can be directly described via Euler-Lagrangian formulation with complex currents. The Lagrangian is the sum of a mechanical kinetic energy and a magnetic Lagrangian. This second term is expressed in terms of rotor angle, complex stator and rotor magnetizing currents. Via simple modification of magnetic Lagrangian we derive a non-trivial dynamical model describing permanent-magnet machines with both saturation and saliency. We propose an experimental validation of such models on a customized torque machine of $1.2 \mathrm{~kW}$. This first validation relies on injections of high frequency oscillations on the stator voltage. According to the proposed saturation model, the resulting amplitudes of the current-ripples is an increasing function of the current offset. Such dependance is effectively observed experimentally and confirmed by simulations.
\end{abstract}

Keywords: Lagrangian with complex coordinates, magnetic saturation, saliency, permanent-magnet machine, high frequency injection.

\section{INTRODUCTION}

Modeling permanent magnet machines with magnitude saturation is not a straightforward task and could lead to complicated developments when a detailed physical description is included (see, e.g.,Chiasson [2005], Boldea and Nasar [2002]). Even if such effects are not dominant they play an important at low speed and/or high torque and stator current. The contributions of this communication are as follows :

- simples models (see (10)), including simultaneously saturation and saliency and extending directly usual models used in the literature.

- A first experimental validation of such magnetic saturation models for a $1.2 \mathrm{~kW}$ machines. It relies on high frequency voltage injection and on the measures of the resulting ripples on the stator current.

We exploit here Basic et al. [2009] that proposes an extension to complex electrical variable of Lagrangian modeling of electrical machines.

In section 2 we recall the simplest model of a permanent magnet machine and its Euler-Lagrange formulation based on the two scalar components of the complex stator current. We recall the complexification procedure and explain how to derive the Euler-Lagrange equations directly with complex stator current. Then we provide the general form of physically consistent models (equation (5)). Finally we obtain, just by simple modification of the magnetic Lagrangian, physically consistent models with magnetic saturation and saliency effects (equation (10)). We also derive the associated magnetic energies of such non-linear magnetic models. In section 3 , we propose a simple experimental validation of the nonlinear magnetic model introduced in previous section (equation (10) with lambda given by (12)). The validation is based on high frequency voltage injection and measurement of the associated currentripples. Perturbations techniques provide analytic expressions of these ripples, expressions depending on the level of permanent currents. Experimental data on a $1.2 \mathrm{~kW}$ machine confirm the fact that the current ripples are an increasing function of the current static offset. These experimental data also confirm that the injected frequencies are not too high (less than a few $\mathrm{kHz}$ ) and that the main flux distribution is not altered by skin effects in magnets and laminations and that magnetic core losses could be neglected here. Simulations confirm that such dependance is typical of such magnetic saturations. 


\section{EULER-LAGRANGE MODELLING WITH COMPLEX CURRENTS AND VOLTAGES}

\subsection{The usual model and its Euler-Lagrange formulation}

In the $(\alpha, \beta)$ frame (total power invariant transformation), the dynamic equations read (see, e.g., Chiasson [2005], Leonhard [1985]):

$$
\left\{\begin{array}{l}
\frac{d}{d t}(J \dot{\theta})=n_{p} \Im\left(\left(\bar{\phi} e^{\jmath n_{p} \theta}\right)^{*} \imath_{s}\right)-\tau_{L} \\
\frac{d}{d t}\left(\lambda \imath_{s}+\bar{\phi} e^{\jmath n_{p} \theta}\right)=u_{s}-R_{s} \imath_{s}
\end{array}\right.
$$

where

- * stands for complex-conjugation, $\jmath=\sqrt{-1}$ and $n_{p}$ is the number of pairs of poles.

- $\theta$ is the rotor mechanical angle, $J$ and $\tau_{L}$ are the inertia and load torque, respectively.

- $\imath_{s} \in \mathbb{C}$ is the stator current, $u_{s} \in \mathbb{C}$ the stator voltage.

- $\lambda=\left(L_{d}+L_{q}\right) / 2$ with inductances $L_{d}=L_{q}>0$ (no saliency here).

- The stator flux is $\phi_{s}=\lambda v_{s}+\bar{\phi} e^{\jmath n_{p} \theta}$ with the constant $\bar{\phi}>0$ representing to the rotor flux due to permanent magnets.

It is well known that (1) derives from a variational principle (see, e.g.,Ortega et al. [1998]) and thus can be written as Euler-Lagrange equations with source terms corresponding to energy exchange with the environment. Consider the additional complex variable $q_{s} \in \mathbb{C}$ defined by $\frac{d}{d t} q_{s}=\imath_{s}$. The Lagrangian associated to this system is the sum of the mechanical kinetic Lagrangian $\mathcal{L}_{c}$ and magnetic one $\mathcal{L}_{m}$ defined as follows:

$$
\mathcal{L}_{c}=\frac{J}{2} \dot{\theta}^{2}, \quad \mathcal{L}_{m}=\frac{\lambda}{2}\left|\imath_{s}+I_{m} e^{\jmath n_{p} \theta}\right|^{2}
$$

where $I_{m}=\bar{\phi} / \lambda>0$ is the permanent magnetizing current.

Take the complete Lagrangian $\mathcal{L}=\mathcal{L}_{c}+\mathcal{L}_{m}$ as a real function of the generalized coordinates $q=\left(\theta, q_{s \alpha}, q_{s \beta}\right)$ and generalized velocities $\dot{q}=\left(\dot{\theta}, \imath_{s \alpha}, \imath_{s \beta}\right)$ :

$$
\begin{aligned}
\mathcal{L}(q, \dot{q}) & =\frac{J}{2} \dot{\theta}^{2} \\
& +\frac{\lambda}{2}\left(\left(\imath_{s \alpha}+I_{m} \cos n_{p} \theta\right)^{2}+\left(\iota_{s \beta}+I_{m} \sin n_{p} \theta\right)^{2}\right)
\end{aligned}
$$

with $q_{s}=q_{s \alpha}+\jmath q_{s \beta},\left(q_{s \alpha}\right.$ and $q_{s \beta}$ real $)$ and $\dot{q}_{s}=\imath_{s}=\imath_{s \alpha}+$ $\imath_{s \beta},\left(\imath_{s \alpha}\right.$ and $\imath_{s \beta}$ real). Then the mechanical equation in (1) reads

$$
\frac{d}{d t}\left(\frac{\partial \mathcal{L}}{\partial \dot{\theta}}\right)-\frac{\partial \mathcal{L}}{\partial \theta}=-\tau_{L}
$$

where $-\tau_{L}$ corresponds to the energy exchange through the mechanical load torque. Similarly, the real part of complex and electrical equation in (1) reads

$$
\frac{d}{d t}\left(\frac{\partial \mathcal{L}}{\partial \dot{q}_{s \alpha}}\right)-\frac{\partial \mathcal{L}}{\partial q_{s \alpha}}=u_{s \alpha}-R_{s} \imath_{s \alpha}
$$

and its imaginary part

$$
\frac{d}{d t}\left(\frac{\partial \mathcal{L}}{\partial \dot{q}_{s \beta}}\right)-\frac{\partial \mathcal{L}}{\partial q_{s \beta}}=u_{s \beta}-R_{s} \imath_{s \beta}
$$

since $\frac{\partial \mathcal{L}}{\partial q_{s \alpha}}=\frac{\partial \mathcal{L}}{\partial q_{s \beta}}=0$ and $\dot{q}_{s}=\imath_{s}$. The energy exchanges here are due to the power supply through the voltage $u_{s}$ and also to dissipation and irreversible phenomena due to stator resistance represented by the Ohm law $-R_{s} \imath_{s}$.

\subsection{Euler-Lagrange equation with complex current}

The drawback of such Lagrangian formulation is that we have to split into real and imaginary parts the generalized coordinates associated to $q_{s}$ and $\dot{q}_{s}=\imath_{s}$. We do not preserve the elegant formulation of the electrical part through complex variables and equations. We will show here that it is still possible to extend such complex formulation to the Euler-Lagrange equations. It seems that it has never been used for electrical machines. We recall here below the principle of such complexification (usual in quantum electro-dynamics) and then applied it to the above Euler-Lagrange formulation.

Consider a Lagrangian system with two generalized coordinates $q_{1}$ and $q_{2}$ corresponding to a point $q=q_{1}+\jmath q_{2}$ in the complex plane $(\jmath=\sqrt{-1})$. The Lagrangian $\mathcal{L}\left(q_{1}, q_{2}, \dot{q}_{1}, \dot{q}_{2}\right)$ is a real function and the Euler-Lagrange equations are

$$
\frac{d}{d t}\left(\frac{\partial \mathcal{L}}{\partial \dot{q}_{1}}\right)-\frac{\partial \mathcal{L}}{\partial q_{1}}=0, \quad \frac{d}{d t}\left(\frac{\partial \mathcal{L}}{\partial \dot{q}_{2}}\right)-\frac{\partial \mathcal{L}}{\partial q_{2}}=0 .
$$

Using the complex notation $q$, we have $q_{1}=\frac{q+q^{*}}{2}$ and $q_{2}=\frac{q-q^{*}}{2 \jmath}$, thus $\mathcal{L}$ is also a function of $q, q^{*}, \frac{d}{d t} q$ and $\frac{d}{d t} q^{*}$ :

$$
\tilde{\mathcal{L}}\left(q, q^{*}, \dot{q}, \dot{q}^{*}\right) \equiv \mathcal{L}\left(\frac{q+q^{*}}{2}, \frac{q-q^{*}}{2 \jmath}, \frac{\dot{q}+\dot{q}^{*}}{2}, \frac{\dot{q}-\dot{q}^{*}}{2 \jmath}\right) .
$$

The above identity defines $\tilde{\mathcal{L}}$ as a function of the 4 complex independent variables $\left(q, q^{*}, \dot{q}, \dot{q}^{*}\right)$. Simple computations show that

$$
2 \frac{\partial \tilde{\mathcal{L}}}{\partial q}=\frac{\partial \mathcal{L}}{\partial q_{1}}-\jmath \frac{\partial \mathcal{L}}{\partial q_{2}}, \quad 2 \frac{\partial \tilde{\mathcal{L}}}{\partial q^{*}}=\frac{\partial \mathcal{L}}{\partial q_{1}}+\jmath \frac{\partial \mathcal{L}}{\partial q_{2}}
$$

and similarly

$$
2 \frac{\partial \tilde{\mathcal{L}}}{\partial \dot{q}}=\frac{\partial \mathcal{L}}{\partial \dot{q}_{1}}-\jmath \frac{\partial \mathcal{L}}{\partial \dot{q}_{2}}, \quad 2 \frac{\partial \tilde{\mathcal{L}}}{\partial \dot{q}^{*}}=\frac{\partial \mathcal{L}}{\partial \dot{q}_{1}}+\jmath \frac{\partial \mathcal{L}}{\partial \dot{q}_{2}} .
$$

Thus with this complex notation, we can gather the two real Euler-Lagrange equations into a single complex one

$$
\frac{d}{d t}\left(\frac{\partial \mathcal{L}}{\partial \dot{q}_{1}}+\jmath \frac{\partial \mathcal{L}}{\partial \dot{q}_{2}}\right)=\frac{\partial \mathcal{L}}{\partial \dot{q}_{1}}+\jmath \frac{\partial \mathcal{L}}{\partial \dot{q}_{2}}
$$

that reads now simply

$$
\frac{d}{d t}\left(2 \frac{\partial \tilde{\mathcal{L}}}{\partial \dot{q}^{*}}\right)-2 \frac{\partial \tilde{\mathcal{L}}}{\partial q^{*}}=0
$$

Let us apply this complexification procedure to the Lagrangian $\mathcal{L}\left(\theta, q_{s \alpha}, q_{s \beta}, \dot{\theta}, \dot{q}_{s \alpha}, \dot{q}_{s \beta}\right)$ defined in (3). The complexification process only applies to $q_{s}$ and $\dot{q}_{s}=\imath_{s}$ by considering $\mathcal{L}$ as a function of $\left(\theta, q_{s}, q_{s}^{*}, \dot{\theta}, \imath_{s}, \imath_{s}^{*}\right)$ :

$$
\mathcal{L}\left(\theta, \dot{\theta}, \imath_{s}, \imath_{s}^{*}\right)=\frac{J}{2} \dot{\theta}^{2}+\frac{\lambda}{2}\left(\imath_{s}+I_{m} e^{\jmath n_{p} \theta}\right)\left(\imath_{s}^{*}+I_{m} e^{-\jmath n_{p} \theta}\right) .
$$

Then the usual equations (1) read

$$
\frac{d}{d t}\left(\frac{\partial \mathcal{L}}{\partial \dot{\theta}}\right)=\frac{\partial \mathcal{L}}{\partial \theta}-\tau_{L}, \quad 2 \frac{d}{d t}\left(\frac{\partial \mathcal{L}}{\partial \imath_{s}^{*}}\right)=u_{s}-R_{s} \imath_{s}
$$

since $\frac{\partial \mathcal{L}}{\partial q_{s}^{*}}=0$ and $\frac{\partial \mathcal{L}}{\partial \dot{q}_{s}^{*}}=\frac{\partial \mathcal{L}}{\partial \imath_{s}^{*}}$.

More generally, the magnetic Lagrangian $\mathcal{L}_{m}$ is a real value function of $\theta, \imath_{s}$ and $\imath_{s}^{*}$ that is $\frac{2 \pi}{n_{p}}$ periodic versus $\theta$. Thus 
any Lagrangian $\mathcal{L}_{\mathrm{PM}}$ representing a 3 -phases permanent magnet machine admits the following form

$$
\mathcal{L}_{\mathrm{PM}}=\frac{J}{2} \dot{\theta}^{2}+\mathcal{L}_{m}\left(\theta, \imath_{s}, \imath_{s}^{*}\right)
$$

Consequently, any model (with saliency, saturation, spaceharmonics, ...) of permanent magnet machines admits the following structure:

$$
\frac{d}{d t}(J \dot{\theta})=\frac{\partial \mathcal{L}_{m}}{\partial \theta}-\tau_{L}, \quad \frac{d}{d t}\left(2 \frac{\partial \mathcal{L}_{m}}{\partial \imath_{s}^{*}}\right)=u_{s}-R_{s} \imath_{s}
$$

and we recover the usual equation with $\phi_{s}=2 \frac{\partial \mathcal{L}_{m}}{\partial \imath_{s}^{*}}$ corresponding to the stator flux. The model considered here above derives from a magnetic Lagrangian of the form

$$
\mathcal{L}_{m}=\frac{\lambda}{2}\left|\imath_{s}+I_{m} e^{\jmath n_{p} \theta}\right|^{2}
$$

with $\lambda$ and $I_{m}$ are two positive parameters.

Many other formulations of $\mathcal{L}_{m}$ are possible and depend on particular modeling issues. Usually, the dominant part of $\mathcal{L}_{m}$ will be of the form $\frac{\bar{\lambda}}{2}\left|\imath_{s}+I_{m} e^{\jmath n_{p} \theta}\right|^{2}\left(\bar{\lambda}, I_{m}\right.$ positive constants) to which is added corrections terms that are "small" scalar functions of $\left(\theta, \imath_{s}, \imath_{s}^{*}\right)$.

The magnetic energy $H_{m}$ does not coincides with $\mathcal{L}_{m}$. It is given by the Hamiltonian, $H_{m}$, defined via a Legendre transform on $\mathcal{L}_{m}$. Following the complex formulation used in quantum electro-dynamics (see [Cohen-Tannoudji et al., 1989, page 88, equation (A.30)]) we have:

$H_{m}\left(\theta, \imath_{s}, \imath_{s}^{*}\right)=\imath_{s} \frac{\partial \mathcal{L}_{m}}{\partial \iota_{s}}\left(\theta, \imath_{s}, \imath_{s}^{*}\right)+\imath_{s}^{*} \frac{\partial \mathcal{L}_{m}}{\partial \imath_{s}^{*}}\left(\theta, \imath_{s}, \imath_{s}^{*}\right)-\mathcal{L}_{m}\left(\theta, \imath_{s}, \imath_{s}^{*}\right)$

Notice that, when $\mathcal{L}_{m}=\frac{\lambda}{2}\left|\imath_{s}+I_{m} e^{j n_{p} \theta}\right|^{2}$ with $\lambda$ and $I_{m}$ constant, we get $H_{m}=\frac{\lambda}{2}\left(\left|\imath_{s}\right|^{2}-I_{m}^{2}\right)$ and we recover the usual magnetic energy $\frac{\lambda}{2}\left|\imath_{s}\right|^{2}$ up to the constant magnetizing energy $\frac{\lambda}{2} I_{m}^{2}$. In next two sub-sections, we introduce some modifications to this standard Lagrangian $\mathcal{L}_{m}$ to take into account saliency and saturation effects and derive the corresponding magnetic energy $H_{m}$.

\subsection{Saliency models}

Adding to $\mathcal{L}_{m}$ the correction $-\frac{\mu}{2} \Re\left(\imath_{s}^{2} e^{-2 \jmath n_{p} \theta}\right)$ with $|\mu|<\lambda$ $(\Re$ means real part) provides a simple way to represent saliency phenomena while the dominant part of the magnetic Lagrangian (and thus of the dynamics) remains attached to $\frac{\lambda}{2}\left|\imath_{s}+I_{m} e^{\jmath n_{p} \theta}\right|^{2}$. With magnetic Lagrangian of the form

$$
\begin{aligned}
\mathcal{L}_{m}=\frac{\lambda}{2}\left(\imath_{s}+I_{m} e^{\jmath n_{p} \theta}\right) & \left(\imath_{s}^{*}+I_{m} e^{-\jmath n_{p} \theta}\right) \\
& -\frac{\mu}{4}\left(\left(\imath_{s}^{*} e^{\jmath n_{p} \theta}\right)^{2}+\left(\imath_{s} e^{-\jmath n_{p} \theta}\right)^{2}\right)
\end{aligned}
$$

where $\lambda=\left(L_{d}+L_{q}\right) / 2$ and $\mu=\left(L_{q}-L_{d}\right) / 2$ (inductances $L_{d}>0$ and $\left.L_{q}>0\right)$, equations $(5)$ become $\left(\lambda I_{m}=\bar{\phi}\right)$

$$
\left\{\begin{array}{l}
\frac{d}{d t}(J \dot{\theta})=n_{p} \Im\left(\left(\lambda \imath_{s}^{*}+\bar{\phi} e^{-\jmath n_{p} \theta}-\mu \imath_{s} e^{-2 \jmath n_{p} \theta}\right) \imath_{s}\right)-\tau_{L} \\
\frac{d}{d t}\left(\lambda \imath_{s}+\bar{\phi} e^{\jmath n_{p} \theta}-\mu \imath_{s}^{*} e^{2 \jmath n_{p} \theta}\right)=u_{s}-R_{s} \imath_{s}
\end{array}\right.
$$

and we recover the usual model with saliency effect. In this case the magnetic energy $H_{m}=\imath_{s} \frac{\partial \mathcal{L}_{m}}{\partial \imath_{s}}+\imath_{s}^{*} \frac{\partial \mathcal{L}_{m}}{\partial \imath_{s}^{*}}-\mathcal{L}_{m}$ is given by:

$$
H_{m}=\frac{\lambda}{2}\left(\left|\imath_{s}\right|^{2}-I_{m}^{2}\right)-\frac{\mu}{4}\left(\left(\imath_{s}^{*} e^{\jmath n_{p} \theta}\right)^{2}+\left(\imath_{s} e^{-\jmath n_{p} \theta}\right)^{2}\right)
$$

\subsection{Saturation and saliency models}

We can also take into account magnetic saturation effects, i.e., the fact that inductances depend on the currents. Let us assume that only the mean inductance $\lambda$ in (7) depends on the modulus of $\imath_{s}+I_{m} e^{\jmath n_{p} \theta}$ and that $\mu$ remains constant:

$$
\lambda=\lambda\left(\left|\imath_{s}+I_{m} e^{\jmath n_{p} \theta}\right|\right)=\lambda\left(\sqrt{\left(\imath_{s}+I_{m} e^{j n_{p} \theta}\right)\left(\imath_{s}^{*}+I_{m} e^{-j n_{p} \theta}\right)}\right) .
$$

The derivative of $\lambda$ versus the modulus of $\imath_{s}+I_{m} e^{n_{p} \theta}$ is denoted by $\lambda^{\prime}$. The magnetic Lagrangian now reads

$$
\begin{aligned}
\mathcal{L}_{m}=\frac{\lambda\left(\left|\imath_{s}+I_{m} e^{\jmath n_{p} \theta}\right|\right)}{2}\left|\imath_{s}+I_{m} e^{\jmath n_{p} \theta}\right|^{2} \\
\quad-\frac{\mu}{4}\left(\left(\imath_{s}^{*} e^{\jmath n_{p} \theta}\right)^{2}+\left(\imath_{s} e^{-\jmath n_{p} \theta}\right)^{2}\right) .
\end{aligned}
$$

The dynamics is given by (5) with such $\mathcal{L}_{m}$. Since

$$
\frac{\partial \lambda}{\partial \theta}=n_{p} \frac{\Im\left(I_{m} e^{-\jmath n_{p} \theta} \imath_{s}\right)}{\left|\imath_{s}+I_{m} e^{\jmath n_{p} \theta}\right|} \lambda^{\prime}, \quad \frac{\partial \lambda}{\partial \imath_{s}^{*}}=\frac{\imath_{s}+I_{m} e^{\jmath n_{p} \theta}}{2\left|\imath_{s}+I_{m} e^{\jmath n_{p} \theta}\right|} \lambda^{\prime}
$$

we get the following model structure with both saliency and magnetic saturation effects:

$$
\left\{\begin{array}{l}
\frac{d}{d t}(J \dot{\theta})=n_{p} \Im\left(\left(\Lambda\left(\imath_{s}^{*}+I_{m} e^{-\jmath n_{p} \theta}\right)-\mu \imath_{s} e^{-2 \jmath n_{p} \theta}\right) \imath_{s}\right)-\tau_{L} \\
\frac{d}{d t}\left(\Lambda\left(\imath_{s}+I_{m} e^{\jmath n_{p} \theta}\right)-\mu \imath_{s}^{*} e^{2 \jmath n_{p} \theta}\right)=u_{s}-R_{s} \imath_{s}
\end{array}\right.
$$

where $\Lambda=\lambda+\frac{\left|\imath_{s}+I_{m} e^{\jmath n_{p} \theta}\right|}{2} \lambda^{\prime}$. It is interesting to compute the magnetic energy ${ }^{2} H_{m}$ from general formula (6):

$$
\begin{aligned}
H_{m}= & \frac{\lambda+\left|\imath_{s}+I_{m} e^{\jmath n_{p} \theta}\right| \lambda^{\prime}}{2}\left|\imath_{s}\right|^{2} \\
& +\frac{\left|\imath_{s}+I_{m} e^{\jmath n_{p} \theta}\right| \lambda^{\prime}}{2} I_{m} \Re\left(\imath_{s} e^{-\jmath n_{p} \theta}\right) \\
& -\frac{\lambda}{2} I_{m}^{2}-\frac{\mu}{4}\left(\left(\imath_{s}^{*} e^{\jmath n_{p} \theta}\right)^{2}+\left(\imath_{s} e^{-\jmath n_{p} \theta}\right)^{2}\right) .
\end{aligned}
$$

Such magnetic energy formulae are not straightforward but there are a direct consequence of such variational formulation of the dynamics and its setting with complex electrical variables.

We will assume now and in the sequel that $\lambda$ admits the following parametric form:

$$
\lambda(\imath)=2 \lambda_{0} \frac{\sqrt{1+\left(\frac{\imath}{\imath_{\mathrm{sat}}}\right)^{2}}-1}{\left(\frac{\imath}{\imath_{\mathrm{sat}}}\right)^{2}}
$$

with two positive parameters $\lambda_{0}>0$ and $\imath_{\text {sat }}>0$. It yields for $\Lambda$ to the following simple expression:

$$
\Lambda(\imath)=\frac{\lambda_{0}}{\sqrt{1+\left(\imath / \imath_{\mathrm{sat}}\right)^{2}}}
$$

Thus with (13), the model (10) describes simultaneously saliency and saturation with 4 physically meaning positive parameters $\mu, \lambda_{0}, I_{m}$ and $\imath_{\text {sat }}$. We will see that such expressions are well adapted for saturation modelling. 


\section{A FIRST EXPERIMENTAL VALIDATION}

\subsection{Description of the test}

Assume that the rotor is blocked via a mechanical brake at position $\theta=0$. Consider the following voltage inputs

$$
u_{s}(t)=u_{s}^{r}+u_{s}^{a} f(\Omega t)
$$

where

- the complex quantities $u_{s}^{r}$ and $u_{s}^{a}$ are constant;

- the real-value function $\gamma \mapsto f(\gamma)$ is $2 \pi$ periodic with a zero mean, $\int_{0}^{2 \pi} f(\gamma) d \gamma=0$;

- the pulsation $\Omega$ is large (typically around $1 \mathrm{kHz}$ ).

In the sequel $F$ denotes the primitive of $f, \frac{d F}{d \gamma}=f$, that admits a zero mean, $\int_{0}^{2 \pi} F(\gamma) d \gamma=0$. Then the electrical dynamics obey to the following differential implicit equation:

$$
\frac{d}{d t}\left(\Lambda\left(\imath_{s}+I_{m}\right)-\mu \imath_{s}^{*}\right)=u_{s}^{r}+u_{s}^{a} f(\Omega t)-R_{s} \imath_{s}
$$

where $\Lambda$ is given by (13). Set $\imath_{s}^{r}=u_{s}^{r} / R_{s}$ its asymptotically and hyperbolically stable solution when $u_{s}^{a}=0$. For $\Omega$ large enough, the solution of (15) converges towards a small periodic orbits around $\imath_{s}^{r}: \imath_{s}(t)=\imath_{s}^{r}+\delta \imath_{s}(\Omega t)$ where the complex-value function $\gamma \mapsto \delta \imath_{s}(\gamma)$ is $2 \pi$ periodic with zero mean. $\delta \imath_{s}(\Omega t)$ corresponds to the socalled current ripples resulting from the high frequency injection $u_{s}^{a} f(\Omega t)$. The above statement can be rigorously proved by averaging theorem (see, e.g., [Guckenheimer and Holmes, 1983, theorem 4.1.1, page 168]) since the unperturbed system is asymptotically stable (for $|\mu|$ small enough, it is in fact a strict contraction in the sense of Lohmiler and Slotine [1998] for the Euclidian metric on the stator flux in $\mathbb{C}$ ).

Standard asymptotics for $\Omega$ tending to $+\infty$ show that the current ripples $\delta \imath_{s}$ satisfy to the following complex equation:

$$
\begin{array}{r}
\left(\Lambda+\frac{\left|\imath_{s}^{r}+I_{m}\right| \Lambda^{\prime}}{2}\right) \delta \imath_{s}+\left(\frac{\left(\imath_{s}^{r}+I_{m}\right)^{2} \Lambda^{\prime}}{2\left|\imath_{s}^{r}+I_{m}\right|}-\mu\right) \delta \imath_{s}^{*} \\
=\frac{u_{s}^{a}}{\Omega} F(\Omega t)+O\left(\frac{\left|u_{s}^{a}\right|^{2}}{\Omega^{2}}\right)
\end{array}
$$

where $\Lambda$ and its $\imath$-derivative $\Lambda^{\prime}$ are evaluated at $\imath=\mid \imath_{s}+$ $I_{m} \mid$. Assume $u_{s}^{r}$ real, then $\imath_{s}^{r}=u_{s}^{r} / R_{s}$ is real too and the above formulae simplify a little

$$
\begin{aligned}
\left(\Lambda+\frac{\left|\imath_{s}^{r}+I_{m}\right| \Lambda^{\prime}}{2}\right) \delta i_{s} & +\left(\frac{\left|\imath_{s}^{r}+I_{m}\right| \Lambda^{\prime}}{2}-\mu\right) \delta i_{s}^{*} \\
= & \frac{u_{s}^{a}}{\Omega} F(\Omega t)+O\left(\frac{\left|u_{s}^{a}\right|^{2}}{\Omega^{2}}\right) .
\end{aligned}
$$

For $u_{s}^{a}$ real, $\delta \imath_{s}$ is real and given by:

$$
\left(\Lambda+\left|\imath_{s}^{r}+I_{m}\right| \Lambda^{\prime}-\mu\right) \delta \imath_{s}=\frac{u_{s}^{a}}{\Omega} F(\Omega t)+O\left(\frac{\left|u_{s}^{a}\right|^{2}}{\Omega^{2}}\right) .
$$

Using (13), we have

$$
\left(\frac{\lambda_{0}}{\left(1+\frac{\left(i_{s}^{r}+I_{m}\right)^{2}}{i_{\text {sat }}^{2}}\right)^{\frac{3}{2}}}-\mu\right) \delta \imath_{s} \approx \frac{u_{s}^{a}}{\Omega} F(\Omega t) .
$$

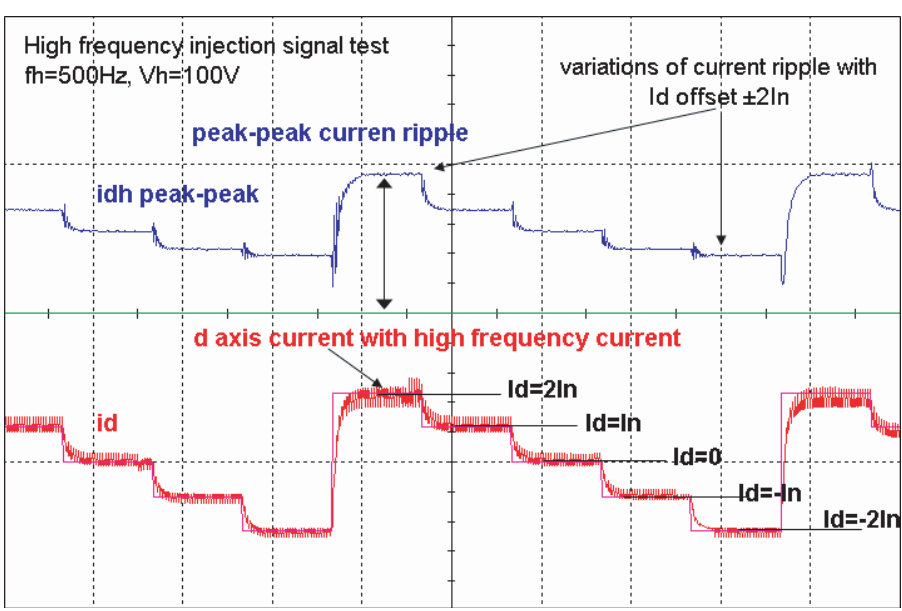

Fig. 1. Experimental data. The blue curve corresponds to the amplitudes of the current-ripples (obtained via a simple PLL-filter); the red curve to the current measures $\imath_{s}(d$-axis aligned with the rotor $\theta=0)$; we observe an increasing dependance of the ripples versus the current offset $\imath_{s}^{r}$.

We should observe experimentally that, for the same high frequency voltage excitation, the amplitudes of the current ripples depend on the current offset $\imath_{s}^{r}$.

\subsection{Simulation vs experimental results for a $1.2 \mathrm{~kW}$ machine}

We take a permanent magnet synchronous motor of $1.2 \mathrm{~kW}$ from the manufacturer Bernecker+Rainer, IndustrieElektonik Ges. M.b.h, Eggelsberg, Austria (reference: 8YS-H0004R0.029-0). For this motor, we have $n_{p}=6$, the nominal current $I_{n}=2.4 \mathrm{~A}$ and $R_{s}=6.7 \mathrm{Ohms}$. The magnetizing current $I_{m}$ is around $2.6 I_{n}$, the saturation current $\imath_{\text {sat }}$ is about $5 I_{n}$, the main inductance $\lambda_{0}$ is equal to $92.6 \mathrm{mH}$ and the saliency $\mu$ almost vanishes. Thus, for this motor and according to (17), the amplitude of the current-ripples should be an increasing function of $\imath_{s}^{r}$ when $\imath_{s}^{r}+I_{m}>0$.

The parameters of the high frequency voltage injection are $\Omega / 2 \pi=500 \mathrm{~Hz}, \quad\left|u_{s}^{a}\right|=100 \mathrm{~V}$ and $\quad f(\gamma)=\operatorname{sign}(\sin (\gamma))$. We use five real values for the constant voltage injections $u_{s}^{r}$ leading to five levels for $\imath_{s}^{r} \in\left\{2 I_{n}, I_{n}, 0,-I_{n},-2 I_{n}\right\}$. For each value of $\imath_{s}^{r}$, we estimate the amplitude of the currentripples via a simple PLL-filter. The experimental results are reported on figure 1.

We observe, as predicted via the theory, that this amplitude decreases when $\imath_{s}^{r}$ decreases. The simulation results are shown in figure 2. This dependance cannot be explained via the standard model (8) with linear inductances and results from non-negligible magnetic saturation effects. Indeed, the simulation results in figure 3, obtained with linear inductances, show constant ripples independently of the total magnetizing current $\left|\imath_{s}+I_{m}\right|$. In the case of the standard model, we define the value of the inductance $\lambda$ by equals to $82.2 \mathrm{mH}$.

$$
\lambda=\frac{\lambda_{0}}{\sqrt{1+\left(I_{m} / \imath_{\mathrm{sat}}\right)^{2}}}
$$



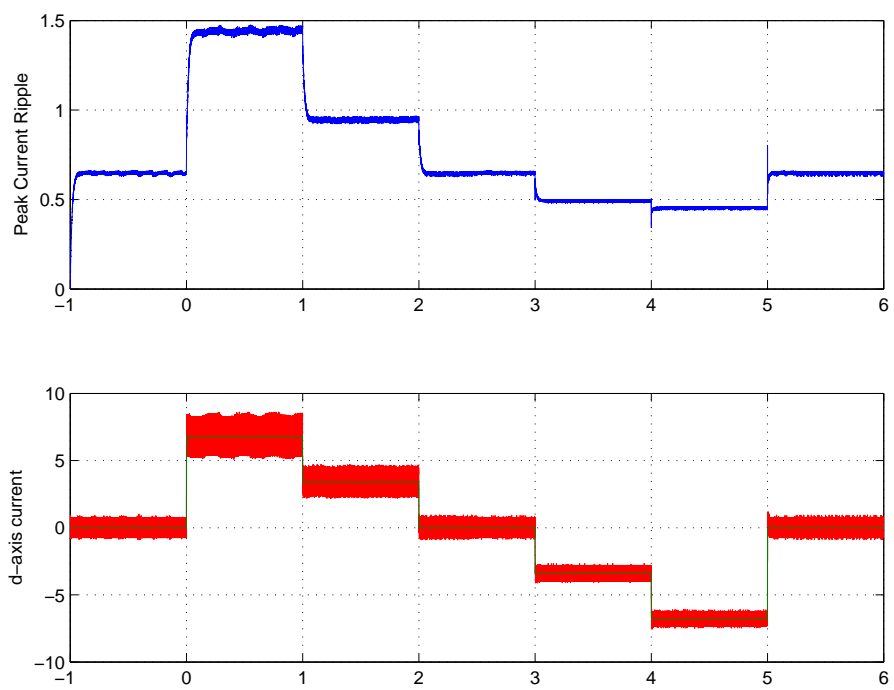

Fig. 2. Simulation data with saturation model. The first curve (up) corresponds to the amplitudes of the current-ripples; the red curve to the current measures $\imath_{s}(d$-axis aligned with the rotor $\theta=0)$; as predicted by theory, we observe in simulation an increasing dependance of the ripples versus the current offset $\imath_{s}^{r}$.
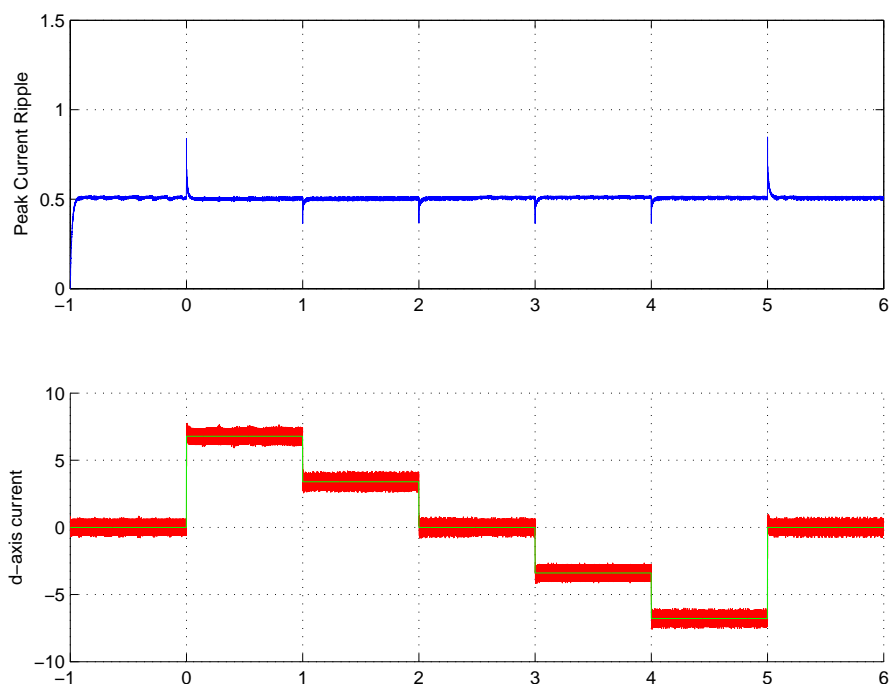

Fig. 3. Simulation data with standard model without saturation. The first curve (up) corresponds to the amplitudes of the current-ripples ; the red curve to the current measures $\imath_{s}$ ( $d$-axis aligned with the rotor $\theta=0$ ); as predicted by theory, we observe in simulation that ripples stay constant independently of the current offset $\imath_{s}^{r}$.

We chose here a particular context with $\theta=0$ in order to get the simplest computation and experimental test of such modelling. The above developments remain also valid when $\theta$ is no more fixed to 0 and when saliency (level and position) induced by saturation depends on the load torque.

\section{CONCLUSION}

The saturation models (10) for permanent-magnet machine with $\lambda$ given by (12) are based on variational prin- ciples and Lagrangian formulation of the dynamics. Experimental data provide a first validation of such modelling procedures that preserve the physical insight while maintaining a synthetic view without describing all the technological and material details.

More complete validations could be done: at non zero rotor velocity, the proposed computations of the current ripples are still possible since they are obtained via usual perturbations techniques. More complex models can be developed with $\mu$ depending also on $\left|\imath_{s}+I_{m}\right|$. Such models can also be used for control purposes: adaptation of usual control schemes to take into account saturation effects are under study.

\section{REFERENCES}

D. Basic, F. Malrait, and P. Rouchon. Euler-lagrange models with complex currents of three-phase electrical machines and observability issues. Accepted in IEEE Trans. Automatic Control, 2009. Preliminary version: arXiv:0806.0387v3 [math.OC].

I. Boldea and S.A. Nasar. The Induction Machine Handbook. CRC Press, 2002.

J. Chiasson. Modeling and High Performance Control of Electric Machines. IEEE Press Series on Power Engineering. Wiley-IEEE Press, 2005.

C. Cohen-Tannoudji, J. Dupont-Roc, and G. Grynberg. Photons and Atoms: Introduction to Quantum Electrodynamics. Wiley, 1989.

J. Guckenheimer and P. Holmes. Nonlinear Oscillations, Dynamical Systems and Bifurcations of Vector Fields. Springer, New York, 1983.

W. Leonhard. Control of Electrical Drives. Elsevier, 1985.

W. Lohmiler and J.J.E. Slotine. On metric analysis and observers for nonlinear systems. Automatica, 34(6):683696, 1998.

R. Ortega, A. Loria, P. J. Nicklasson, and H. SiraRamirez. Passivity-Based Control of Euler-Lagrange Systems. Communications and Control Engineering. Springer-Verlag, Berlin, 1998. 